\title{
Birthplace as the midwife's work place: How does place of birth impact on midwives?
}

Davis DL and Homer CSE, Women and Birth, 2015

\section{Abstract}

Background: In, many high and middle-income countries, childbearing women have a variety of birthplaces available to them including home, birth centres and traditional labour wards. There is good evidence indicating that birthplace impacts on outcomes for women but less is known about the impact on midwives.

Aim: To explore the way that birthplace impacts on midwives in Australia and the United Kingdom.

Method: A qualitative descriptive study was undertaken. Data were gathered through focus groups conducted with midwives in Australia and in the United Kingdom who worked in publicly-funded maternity services and who provided labour and birth care in at least two different settings.

Findings: Five themes surfaced relating to midwifery and place including: 1. practising with the same principles; 2 . creating ambience: controlling the environment; 3. workplace culture: being watched 4 . Workplace culture: "busy work” versus "being with”; and 5. midwives' response to place.

Discussion: While midwives demonstrate a capacity to be versatile in relation to the physicality of birthplaces, workplace culture presents a challenge to their capacity to "be with" women.

Conclusion: Given the excellent outcomes of midwifery led care, we should focus on how we can facilitate the work of midwives in all settings. This study suggests that the culture of the birthplace rather than the physicality is the highest priority.

\section{Keywords}


Midwifery, workplace, parturition, birthplace, workplace culture, environment

\section{Summary of Relevance}

\begin{tabular}{|c|c|}
\hline Problem & $\begin{array}{l}\text { Birthplace has a significant impact on childbearing women but we } \\
\text { know little about how it might impact on midwives. }\end{array}$ \\
\hline $\begin{array}{l}\text { What is Already } \\
\text { Known }\end{array}$ & $\begin{array}{l}\text { Midwifery led care and birth in primary settings result in excellent } \\
\text { outcomes for women though most births in the western word } \\
\text { continue to occur in birthplaces situated in acute care settings. } \\
\text { Midwives are generally dissatisfied with these work environments } \\
\text { from a practical perspective and they understand that the aesthetic } \\
\text { of place impacts on their mood. }\end{array}$ \\
\hline $\begin{array}{l}\text { What this Paper } \\
\text { Adds }\end{array}$ & $\begin{array}{l}\text { Midwives can manage many aspects of the physical environment to } \\
\text { make it more conducive to childbearing women. Creating an } \\
\text { appropriate ambience for birth is recognised as a key feature of } \\
\text { midwifery practice though all those who occupy birthplaces do not } \\
\text { appreciate this. The same environment that is most conducive to } \\
\text { birth is also conducive to safe midwifery practice. }\end{array}$ \\
\hline
\end{tabular}




\section{Introduction}

Childbearing women have a variety of birthplaces available to them including home, birth centres and traditional labour wards situated in high acuity hospitals. There is evidence indicating that planned birthplace impacts on outcomes for childbearing women..$^{1-5}$ Women for whom a normal labour and birth can be anticipated, who plan to give birth at home or in birth centres experience less obstetric intervention and have higher rates of normal birth than comparable women planning to give birth in traditional hospital settings. While there is potential for selection bias in the observational studies that inform this understanding, the findings of research examining the impact of birthplace on childbirth outcomes are robust and remarkably consistent internationally. Women's maternity outcomes vary considerably according to planned place of birth. This means that either the choices and behaviours of childbearing women or the physiology of labour and birth varies in different types of settings (and that these choices, behaviours and physiology impact on their outcomes), and / or the practices of maternity caregivers vary in different settings.

Women who plan to give birth at home or in birth centres usually have a desire for a normal birth but it is difficult to understand how desire alone can impact on birth outcomes. Desire for a normal birth however leads to choices that increase the chances of a normal birth like for instance, waiting for labour to commence spontaneously rather than inducing labour and eschewing pharmacological pain relief in preference for non-pharmacological methods. ${ }^{6}$

Women who labour at home or in birth centres may also behave differently. For example, these settings encourage women to be active during labour whilst the dominance of the obstetric bed in traditional hospital settings invites a more passive response to labour. ${ }^{7}$ In addition, the physiology of labour and birth may be altered by place. Researchers have hypothesised that different places elicit responses in women that can promote or inhibit the 
physiology of birth. The "fear cascade" is initiated by places that induce anxiety and stress, resulting in increased secretion of catecholamines (such as adrenaline), which diminish the secretion of oxytocin thus disrupting the physiological progress of labour. The opposite is true of places that induce feelings of safety and calm. ${ }^{8}$ These issues influence women and probably also the midwives who care for them.

\section{The birthplaces of women are the workplaces of midwives}

The birthplaces of childbearing women are the workplaces of midwives. Workplaces or any place for that matter, are comprised of both tangible and non-tangible elements. The tangible elements include the physical characteristics of the place such as the layout, design and equipment. The non-tangible elements include things like the workplace culture. Both these elements of the workplace have the potential to impact workers. Studies have demonstrated that midwives are affected by the design of maternity settings. ${ }^{9-11}$ Design can affect physical and psychological health and also impact on the ability of midwives to do their job effectively. In an Australian study midwives raised concerns about their ability to care for women in cramped and cluttered spaces, particularly in emergency situations, the difficultly in providing for their own comfort whilst supporting labouring women and occupational health and safety concerns in relation to supporting women using water immersion for labour and birth. Midwives in this study also acknowledged the impact of aesthetics on their own mood.11

Similarly, in a UK study, midwives were generally dissatisfied with their workplaces citing issues of privacy, security, lack of spaces that provide for their own respite and lack of visual access to outdoors as their main concerns ${ }^{9}$. Again in the UK, Symon et al ${ }^{10}$ found that many maternity units were not providing adequate facilities for staff respite, showering and changing. Maternity units varied in relation to the way the layout facilitated observation of mothers and babies and promoted positive staff interaction, with midwifery led units 
scoring more favourably in these areas than obstetric led units. Air quality, lighting and lack of access to rest areas were the most commonly cited factors impacting on personal health.

The non-tangible elements of the workplace also influence midwives in a variety of ways. Non-tangible elements of the workplace include the workplace culture; a nebulous concept but something that nonetheless has a powerful impact on the wellbeing and behaviours of employees. Workplace culture has been defined as “... the shared values, beliefs, assumptions and norms that affect the way people and groups in an organisation interact with each other" (p. 5).12 Workplace culture is comprised of four elements; artefacts, behavioural norms, values and assumptions. Artefacts include symbols and objects that express cultural messages. In the maternity context these might include the equipment or signage visible in a birthing room or the way a room is set up in expectation of a particular type of birth. Behavioural norms are the behaviours and practices that are expected and "allowed" within a workplace. Values are not always those articulated in advertising materials but are most clearly illustrated by the practices, outcomes and priorities encouraged and rewarded by a workplace. While an organisation might state that they value woman centred care they may in fact prioritise and encourage efficiency. Finally, assumptions are the beliefs that underpin all other aspects of workplace culture. In the maternity setting, assumptions may include that birth is inherently risky or that all women will require pain relief in labour. Studies examining midwifery practice in hospital settings suggest that powerful cultural norms shape practice in these settings ${ }^{13}$ and point to issues of power and obstetric dominance, ${ }^{14}$ practices underpinned by the assumption that birth is inherently risky, ${ }^{15}$ and concerns for efficiency over a woman-centred approach to care. ${ }^{16}$

Given the evidence that shows different outcomes for women in different settings and the challenges facing midwives in supporting physiological birth in some settings, it is essential to better understand how different sorts of places (or birth environments) impact on 
midwives. This is particularly so for midwives who work across different settings and therefore may alter their behaviour or approach according to their setting. The aim of this study therefore was to explore the way that different places of birth (home, birth centre, labour ward) impact on the behaviours, emotions and experiences of midwives in Australia and the United Kingdom. It is not the intention of this paper to 'pit' one setting against another but rather to use the comparisons and contrasts of/between settings to highlight aspects of midwifery work that midwives consider important and to consider how this is impacted by place. Given that midwives provide the mainstay of labour and birth care in all birth settings, it is important to understand their experience of place so as to better understand how we can optimise the environment so as to enhance midwifery care in all birthplaces.

\section{Methods}

\section{Design}

A qualitative descriptive study ${ }^{17,18}$ was undertaken to explore the perceptions of midwives who worked across different birth settings in two countries. The relevant Research Ethics Committees approved the study.

\section{Participants and settings}

The participants were midwives who practise concurrently in a variety of settings including traditional labour wards (also known as birth or delivery suites), a birth centre co-located with tertiary maternity services and home as part of publicly funded homebirth services. In this paper home and birth centres are sometimes collectively referred to as "primary birth settings" and traditional labour wards, obstetric led units or birth suites situated in acute care hospital settings are referred to as "labour wards". 
Midwives working in two continuity of midwifery care practices in Australia (sites A and B) and one in the UK (site C) which offered care to women in at least two of the above settings concurrently were invited to participate. Midwives from site A supported women to give birth in a birth centre or at home. If women in their care required obstetric intervention they also continued to provide care in the labour ward. Midwives from site B supported women to give birth at home or in the labour ward of a tertiary level maternity hospital. Midwives from site $\mathrm{C}$ supported women to give birth in a traditional labour ward or at home.

Midwives in these practices were contacted with a request to participate in the research in writing. Twelve midwives in total agreed to participate and provided signed informed consent; five and three from sites A and B respectively in Australia, and four from site C in the UK. Two to four midwives participated in each focus group and while greater numbers were anticipated, this reflects the difficulty in scheduling events with midwives who have on-call commitments. Table 1 shows the number of midwives participating at each site along with their years of experience in midwifery.

[Table 1 about here]

\section{Data collection}

Four focus groups were held with twelve participating midwives from the three sites with each lasting between 45 and 70 minutes. They were held in a variety of locations of the participant's choosing including the workplace and a participant's home. Focus groups are a useful qualitative research technique that exploits the potential for interaction when individuals are brought together in a group situation ${ }^{19}$. It has been recognised that "the group dynamic can assist people in expressing and clarifying their views in ways that are less likely to occur in a one-on-one interview" 20 p. 412 Thus the focus group elicits greater 
depth and breadth of information than may be obtained through other data collection techniques.

Focus groups were facilitated by three female midwife researchers, two of whom were experienced researchers and one of whom was undertaking a research training program under supervision. The research midwives were known to some of the participants though they did not have a close working relationships and they were not in positions of power over the participating midwives. The two experienced researchers were also experienced in supporting women to give birth in different settings and in researching birth environments. Undoubtedly, their experiences and previous research work contributed to the way they conducted this study, this is unavoidable. Having said that, great care was taken in all phases of the research to approach the topic naively and avoid making assumptions or imposing theoretical frameworks or prior knowledge on any aspect of the research.

The facilitator used an interview guide to provide a framework for the discussion, which was followed loosely to allow a more free flowing discussion to emerge. This included open and naïve questions like for example "do you think these different environments impact on your practice in any way?" (rather than "how does the environment impact on your practice?") to more direct questions such as "are there things that you would feel comfortable doing in one setting that you wouldn't in another?" The facilitator used prompts such as "can you tell me why?" and "can you expand on that?" to delve into an issue and questions such as "did anyone else have that experience?" to ascertain the level of consensus or draw out opposing positions. The focus groups were audio recorded and transcribed verbatim. To protect participant confidentiality, all place and person names were replaced with "[name of person/place]" in the transcript. Quotations from the focus groups included in this paper are referenced to the focus group number (1-4). "FG4" therefore refers to focus group number 4. 


\section{Analysis}

Thematic analysis was undertaken by hand, following a qualitative descriptive approach ${ }^{17}$. A qualitative descriptive approach aims for a low-inference analysis whilst acknowledging that no description is free of interpretation, since it must be filtered through human perceptions [15]. The process is inductive as meaning emerges from the data ${ }^{21}$ and the researcher stays close to the data and to the "surface of words and events" 17 p. 334.

Focus group transcripts were read and each unit of meaning (a sentence or group of sentences conveying a concept or message) was provided with a descriptive label. These descriptive labels were examined and converged with similar descriptive labels where this was possible. In addition before deciding on the final themes we ensured that the emerging themes arose from all focus groups. This was achieved by colour coding each focus group transcript and building a table with tentative themes and illustrative quotations. Any comments representing dissenting views were also recorded. The only real point of dissention came from the question "do you think these different environments impact on your practice in any way?" Midwives in 3 focus groups replied emphatically in the positive for example "Most definitely, definitely (quickly)(FG4), while one midwife in focus group 2 responded "I don't know that there is a great deal of difference in my practice because we follow the same policies and procedures and everything else that we do in hospital - it's no different". However this did not impact on the development of the themes as once the topic was delved into further, the comments of this midwife and those of her colleagues in focus group 2 contributed equally to the final themes.

The colour coding allowed us to see where one particular transcript might be making a dominant contribution to a theme. In this case, we re-examined all transcripts to assess whether the theme was represented across all the data and if not, re-considered the theme. Both authors read all transcripts and contributed to the analysis. The first author took a 
lead in developing tables and tentative themes and these formed the basis of discussion and finally after several iterations, agreement between the authors.

\section{Findings}

Participating midwives had between 2-30 years experience in midwifery (with an average of 15 years). Through reflecting on their practice and experiences in different birth settings, the midwives highlighted a number of themes related to the practice of midwifery and the impact of place. These include: practising by the same principles; creating ambience: controlling the environment; workplace culture: "busy work" versus "being with" and being watched and, the midwives' emotional response to place.

\section{Practising with the same principles}

Participating midwives were keen to point out that their practice principles remained the same whatever the care environment. These include monitoring the wellbeing of mother and baby, assessing the progress of labour and providing the necessary care and support to facilitate a safe and satisfying labour and birth. For example:

I feel like my principles, the way that I practice as a clinician is very similar in the three environments that I work in. ... I think the environment impacts the way that I work but the actual skills and techniques and why I do things at different times would be very similar (FG2).

All midwives recognised that they worked to clinical protocols that were consistent for low risk women regardless of the care setting. For example:

I would say that they are very similar but I think the feeling at home is quite different for obvious reasons because ... you're in their environment and ... although we are still following policy, it has just got a very different feel about it (FG2). 
However as will become clear in the following pages, despite working to consistent clinical protocols, practice philosophy and principals the environment had a significant impact on the midwife.

\section{Creating ambience: controlling the environment}

Ambience is a non-tangible element of the birthplace and it is something that has the capacity to influence the way that we feel and act in different environments. Midwives recognised the importance of ambience to the physiological and emotional aspects of childbirth and work hard to create an ambience that is private, calm and reassuring. They described the way that the ambience was important to the "flow of hormones" in the childbearing woman and thus how creating an appropriate ambience for labour and birth is a key feature of their role as midwives.

The capacity to control the environment to create an appropriate ambience varied in different settings. They highlighted aspects of the environment that are important to ambience and these include; noise, lighting, privacy and a domestic rather than clinical character. Home and to some extent birth centres, had the advantage of a prevailing domestic character while labour ward often had to be adjusted to soften or hide the clinical features. Midwives said:

.... all the equipment, they look so medical. It's so clinical that I think that makes them feel like something could go wrong (FG1).

I always close that shutter. There's a shutter at the end of the delivery suite rooms and it's got metal trolleys and things and IV poles [behind it]. I always close that across cause I don't like it. Sometimes there is an equipment cupboard and I always close 
that. I make sure all the medical stuff is kind of hidden and, like I said, I often lower the bed and I dim the lights (FG2).

Birth rooms in the labour ward could be made to look and feel more like birth centres by for example dimming the lights, moving the obstetric bed and using mats on the floor:

I've set up [room number] in the way that we set the space up for a woman, and I've said after [to the woman] "look I'm really sorry it wasn't in the birth centre". And she said, "it was the birth centre as far as I'm concerned, it was just like being in the birth centre". I thought oh that was good (FG1).

Lighting and noise were identified as important elements of ambience with dim lighting and low noise levels being most desirable. These excerpts highlighted the care that is taken to cultivate a suitable ambience and point to the way that a carefully created ambience can easily be lost:

They came in the morning and the toilet door was open just enough so that I could see what I was writing and the atmosphere had been very calm and ... a lovely night, the girl was progressing on and the day shift came on and said 'How on earth can you see. What you are doing in here?' and put the lights on and using a normal voice. She ruined a whole night shift's work in 5 minutes (FG4).

... and then, not listening to everybody else's labour or other noises. So that ambiance is, for a start, a wonderful thing. As hard as they've tried to dim the noise between rooms, it does carry. And if you open the door just to get out to get something, you know, you hear just about everything and you hear staff sitting outside yapping away and talking about this room and telling jokes and all sorts of things. Even if you've 
created that ambiance in the birthing room you've lost the moment, whereas at home it is continuous (FG 3).

Midwives suggested that ambience might influence the "flow of hormones" necessary for women's effective labour and one of the factors impacting on this was privacy. In some settings, midwives were disturbed by the lack of privacy afforded the childbearing women recounting situations where other staff members have entered into birth rooms un-invited illustrated here:

You know "is there an IV pole in here?" They walk in for that. I usually... if they do kindly knock, which they don't always do, I always try to get them to hold on and not let people to come in. But sometimes you can't control it. You are much less likely to be able to control it in delivery suite than here [birth centre] and obviously it's different at homebirth again. Yes, it interrupts things (FG1).

Midwives in hospital settings however did have strategies for achieving privacy and ambience that is more conducive to facilitating the "flow of hormones", for example:

That's right. So it's a bit harder.... I've put couples in the bathroom and both in the bath, soft music in the bathroom, turned the lights off, kicked the mothers out and said, "leave them alone for a while". And the next thing they are ready to have a baby (FG 3).

Home and to some extent birth centres, provided opportunities for women to control their environment whilst this was not always the case in the labour ward: 
We've also had several women now who like to labour, for at least part of their labour, by themselves. So they go off into a bedroom, usually in the dark and want to be by themselves. Now at home they can do that (FG 3).

One midwife said:

Even, I find at the birth centre, women or the birthing family are more likely to move furniture or turn lights out whereas in delivery suite it's like "oh we better not touch anything"

and another agreed with:

Yeah, it's not their space (FG1).

While the midwives felt that home was often the place that provided for a more ideal ambience for birth, this was not universally the case. In this example, home did not provide the sort of environment that the midwife thought was conducive for birth but of course, this does not consider the childbearing woman's perspective on this situation and this is what matters most. The midwife said:

So it was in another place. But then it turns out that multiple people lived at this place. We were there early hours of the morning and it started off alright but then suddenly people started appearing, getting up, making breakfast, coming through the house, children. It was like this tribe of people coming and going. This just feels wrong! The whole place felt wrong. It didn't feel private and it wasn't her house. We just felt from the beginning - I don't think this is going to be successful here. So the environment and the people around ... that ambiance that you create, absolutely affects birth (FG 3). 
In relation to ambience and the creation of a space that was conducive to birth, the work of midwives in hospital and primary birth settings differed. Home does not always provide the ideal environment for birth but, in general, midwives have to work harder to create and maintain a suitable space for birth in the hospital setting. For example:

You have to work really hard to make them [childbearing women] more relaxed than you do at home, much, much harder (FG1).

It's difficult in delivery suite [traditional labour ward] because there is a culture there that it's not the woman's space, it's everyone's space and I think that's what we have to be really mindful about. And you are spending a lot of time trying to ... protecting her space and I find that exhausting. It takes you away from what you want to be doing and that is hopefully not too much, but just so that she can just do what she needs to do (FG1).

Ambience and the creation of a space that is conducive to labour and birth was described as an important part of midwifery practice. Home and birth centres have the advantage of an existing domestic rather than clinical character and in these settings midwives could more easily provide for privacy. Women and their partners and supporters were more able to control aspects of the environment contributing to the overall ambience at home and to some extent in birth centres. An appropriate ambience for birth can be created in any setting though the hospital provides greater challenges. The midwifery role of creating and maintaining a suitable ambience for birth was impacted by place. 


\section{Workplace culture: "busy work" versus "being with"}

Workplace culture embodies the unwritten rules of a workplace, which include behavioural norms for both childbearing women and midwives. This research identified "busy work" as a behavioural norm or expectation of midwives in labour ward settings. Midwives were expected to "look busy" and attend a variety of additional tasks that often draw their focus away from the childbearing woman in this place. In contract, when practising at home or in a birth centre, midwives were able to engage in behaviours that would be considered "unprofessional" in the hospital setting like enjoying a cup of tea with the family, taking off their shoes and resting the couch. These behaviours however contributed to a sense of conviviality that was so important to the ambience of the environment,

The expectation for "busy work" in the hospital setting means that midwives do not feel comfortable just "being with" women in ways that are acceptable in other settings such as home and birth centres as illustrated by:

I feel like I am just waiting, just watching, not having to do that busy work that sometimes... (FG1).

.... you don't get caught up with the busy work. There is nothing else to do (FG1).

You feel obliged to look busy in the birthing area whereas at home you wouldn't (FG 3).

... and that's expected of you. When you walk in you will check the resus trolley, check equipment, set up your delivery set, and restock anything you need in the room (FG 3). 
"Busy work" prevented midwives from sitting quietly with women and creating the calm and relaxing environment that they described as being so important to the birthing room ambience. Importantly, while midwives worked hard to create this ambience for the women for whom they are caring, midwives also seem to benefit greatly by feeling more relaxed and less stressed in these environments.

Midwives were keenly aware of the behavioural norms for the different settings and disciplined their behaviour accordingly. For example:

When I was delivering a girl, I would be saying have a cup of tea and I would have a cup of tea. You are still giving her the best care and things but it just felt more relaxed and like a nice happy occasion but you can't sit (in hospital) with your wee pot of tea, no, (laughter). Can you imagine!! (FG4).

I wouldn't dream of reading a book or doing a crossword in the birthing area. You might do that in a woman's home because you don't feel obliged to do stuff (FG 3).

Referring to supporting women at home these midwives highlighted the way they could focus on the woman in the home environment. This is an issue of safety for the midwife represented in the second excerpt:

It's only geography as far as I am concerned but for me as a midwife it's calmer, more pleasant, more enjoyable. And I am not focused on doing a thousand other things. I am more focused on the job at hand and that is looking after the woman and the family (FG 3). 
But I found hospitals are less safe than home, but that's my opinion because at home you are totally more focused on the woman and you are hers completely. At the hospital you tend to be diverted, get busy with other things. Your focus isn't on that person and so you miss things - you do miss things and things happen very quickly during labour and birth. But at home you are totally focused on that family and the woman who is birthing (FG 3).

"Being with" childbearing women did not necessarily mean a constant and intense presence but a readiness and availability to provide more hands on support or guidance when needed. Despite the range of "leisure" (such as, reading) or domestic (such as, making a cup of tea) activities in which midwives might be engaging at home or in birth centres, they maintained a vigilance and awareness of the woman and her labour progress, "I am always watchful - much more guided by the woman and what she's wanting and what she's doing" (FG1).

The capacity of midwives to "be with" women in these ways was also confounded by the model of care in which the midwife was working. Midwives in this study were working in continuity models of care and they realised that this provided them with unique opportunities for providing one to one labour care, that were not afforded to midwives working in traditional fragmented models of care. They acknowledged the heavy workload of midwives in labour wards. They often contributed to the work of these midwives when they were in the labour ward environment even though this drew them away from the childbearing woman in their own care as described here:

I have no problems sitting down and reading a book beside a labouring woman or just sitting there and things. I could not do that in the birthing area. I would feel that I 
needed to go and fill something up or restock something. I would feel guilty having a cup of tea if my colleagues were busily running around (FG 3).

Midwifery practice was shaped by powerful cultural norms in different settings. It is appropriate for midwives to engage in domestic activities or other activities in home and birth centre settings while these would be considered unprofessional in the hospital labour ward. Midwives feel guilty providing one to one care to women in the labour ward when they know that their colleagues are busy so they often assist the labour ward midwives where they can, though this prevents them from being with the woman for whom they are providing care. The midwifery role of being with women is impacted by place.

\section{Workplace culture: being watched}

Midwives participating in this study felt that they do not exercise the same degree of autonomy and work according to their full scope of practice which included facilitating births and providing care to the woman and her newborn infant on their own responsibility when supporting women to give birth in traditional labour wards compared to primary birth settings. They felt they were 'being watched' in the labour wards. In the community or birth centre they were trusted to work to their scope of practice and communicate with colleagues if there were concerns. For example, it would be unusual for labour ward staff to contact midwives supporting women at home for updates on the woman's progress however this was frequently the case when the same midwives provided care to women in the labour ward setting. For example:

But in the birthing unit, depending on who is on, you often get people who want to know everything you're doing. Other staff members, they have to know what's going on. And that's fair enough if they're in charge of that shift they need to know, but to the point where you are not saying "I trust these midwives to do what they do best and I 
can just sit back and they'll tell me if there's a problem". And that irritates me because somebody doesn't trust me enough to let me get on with my job and know when to interfere or not to (FG 3).

You're very much an autonomous practitioner in community and you do your own thing and you are left to manage although if you have problems you have support, whereas if you are in the hospital environment especially in the labour ward there tend to be people at your back looking over your shoulder all of the time (FG4).

This last excerpt refers to a sense that their midwifery practice was under continuous scrutiny in the labour ward setting and this was a common sentiment amongst all participants. This had the effect of unsettling and undermining the confidence of these midwives in this place; "Yeah, if you find you're being watched then certainly you don't feel as confident" (FG2).

In the labour ward environment, midwives often feel pressure to ensure that women progress within obstetric timeframes, as expressed here:

But you still have to abide by the guidelines. You still have to do that. I think you are just rushing them all the time in the hospital; you have to do this by this time and this by that time (FG4).

If everything is going normally it is just ... you're guided by the woman. In a delivery suite [labour ward] I feel that there's timeframes and time limits. You know... if this doesn't happen by this time they are going to be knocking on the door going "well what's going on?" (FG1). 
Midwives used more personalised criteria for assessing labour progress that was not based on timeframes alone. They clearly understand the parameters of normal though a more flexible definition of normal was employed:

... I think that you challenge yourself with the variations of normal in the birth centre whereas in the delivery suite you tend to get caught up with the obstetric philosophy (FG1.)

You aren't going against policy. You know this is a normal process and you know she's going to keep going but because you're in a hospital environment you are on the edge rather than [feeling comfortable to] let them get to that point to achieve a natural birth (FG3).

The dominance of obstetric practice frameworks in the labour ward environment and the surveillance of midwifery practice impacted on midwifery practice. Midwives were able to monitor labour progress in ways that were more nuanced and personalised in birth centres and at home. They felt that this helped women to achieve a normal birth and reduce the stress experienced by midwives and women related to the pressure to conform to obstetric parameters of progress that are heavily time dependent. Midwifery autonomy, especially the ability to monitor progress and work to midwifery frameworks was impacted by place.

\section{Midwives' response to place}

Place has a powerful effect on people and both the tangible and intangible elements of place contribute to this. Midwives in this study acknowledged that while birth happened similarly, while they utilised many of the same skills and indeed worked to the same clinical policies in the different places they supported women to give birth, these places had a 
different "feel" and that they felt different in these different places. They expressed this by saying:

I would say that they are very similar but I think the feeling at home is quite different for obvious reasons because ... you're in their environment and ... although we are still following policy, it has just got a very different feel about it (FG 2).

Birth centre and home environments were described as calm and relaxing, "Calm. You just feel so much more relaxed". Despite the fact that birth in primary settings lacks proximity to emergency back up, midwives described feeling more relaxed in primary settings:

But it does sound an odd comment to make on reflection, what I've just said, because it makes it sound like I am less stressed on my own when I am totally isolated environment than I am with someone, a qualified midwife from delivery suite next to me. But that's the reality (FG 2).

I am much more relaxed in a woman's home. It's funny because really I've less backup ifyou think about it, but it is much more relaxing being in a woman's home. (FG1).

In contrast, labour wards were described as stressful environments that significantly impacted on the mood and practice of midwives; "I would say that has a very, very significant impact on my practice because I just find it such a very stressful environment" (FG2). Some of the factors contributing to the stress of labour ward environment included the sense of being watched, "I would feel more relaxed without the watching". The domestic setting and the domestic activities engaged in by the midwives at home and in birth centres contributed to this response: 
You curl your feet up on the lounge and you curl up into one corner or on the chair somewhere or a beanbag or wherever we are and pick up a book and you wander off and make a cup of tea (FG 3).

These settings felt "normal" to midwives and this contributed to a feeling of relaxation.

Participant 2: You know what, it makes us feel normal.

Participant 3: You're in their house doing normal everyday activities. We've folded washing, put the washing on (FG 3).

More relaxed, more family friendly, more... its like being part of a normal thing (FG4).

Normal was opposed to "clinical". In labour ward settings, the artefacts of obstetrics and interventionist birth contributed to the clinical ambience as explained here:

Drip stands [are] everywhere and a CTG [fetal monitor] in every room. A drip stand with an epidural pump and two regular pumps. The resuscitaire used to have doors on it, the doors [have been] taken off... So that we've gone to this much more clinical environment. Now we've got signs on the walls that in the event of an emergency press this button, do this, call this [number]. So you've got these warning signs everywhere (FG 3).

In this environment midwives respond by going into what they called "clinical mode":

P3: Those things work against trying to create that ambiance.

P2: You go into clinical mode don't you? (FG 3)

Midwives felt more stressed and less relaxed in the labour ward compared to birth centre and home environments. In environments that are more "normal" and less clinical, 
midwives could engage in everyday activities whilst maintaining a watchful eye on the childbearing woman. Domestic settings and the workplace culture of primary birth settings (including home) allowed them to be with women in ways that contributed to a calm and relaxing ambience. These environments promoted relaxation for the childbearing woman and also for the midwives. This clearly shows that place has an impact on midwives emotional responses to their work and this likely played out into their behaviours and practices.

\section{Discussion}

Midwives participating in this study acknowledged that place, significantly shapes their practice and the way they feel. It is interesting that the tangible elements of the environment were barely mentioned by midwives, only insofar as they contribute to the "feel" or ambience of the birth space. Midwives perceived greater autonomy in primary birth settings and fewer pressures to conform to workplace norms that privilege "busy work" over "being with" women. In primary birth settings midwives have greater capacity to create an ambience that they feel is conducive to birth. In primary birth settings, these factors combine to create an environment that is less stress inducing for midwives.

Midwifery autonomy reflects the degree to which midwives can make their own practice based decisions. High-level evidence indicates that midwifery led care is a safe (and in many ways superior) model for childbearing women resulting in good clinical outcomes. ${ }^{22} \mathrm{We}$ therefore have good evidence to support midwifery autonomy though despite this, many maternity services continue to be dominated by obstetrics. In obstetric led services midwives may not be able to exercise the freedom to make their own clinical decisions and rather, are directed in their care by obstetricians or obstetric policies. Obstetric dominance and the lack of midwifery autonomy were themes identified by $0^{\prime}$ Connell, Downe ${ }^{13}$ in a meta synthesis of 14 qualitative studies conducted in five countries, exploring midwives' 
experience of hospital practice in public maternity services. In our study, the lack of midwifery autonomy, the pressure to ensure that women conformed to obstetric timeframes and the feeling that someone is always "watching over your shoulder" are significant sources of stress for midwives. These midwives did not experience the same level of scrutiny over their practice when they were practicing as part of the same service in a different setting, which suggests that this type of surveillance has become a cultural norm (rather than a clinical imperative) in these settings. The surveillance of midwifery practice and the imposition of obstetric clinical practice norms in the labour ward environment are not justified given the poorer outcomes that obstetric led services achieve, particularly for women at low risk of experiencing complications.

Another important aspect of place was the way place shapes the ability of midwives to "be with" women. In the labour ward, strong cultural norms press midwives to engage in "busy work" while they feel more able to sit quietly with women in primary birth settings. Midwives are more able to focus on the woman and her family in primary birth settings while "busy work" fragments their focus; an issue of safety for some. Today's labour wards are busy places no doubt and midwives participating in this study recognise that their labour ward colleagues have to work hard, often without opportunities to provide one to one care to labouring women. However, privileging "busy-ness" and "doing" over being with women in ways that promote stillness and calm may be a false economy especially if the ambience created by "busy-ness' disrupts the physiology of the labouring women or the focus of the midwife. Midwives have long recognised the value of the "art of doing nothing well" 23 p.12 and "doing less to give more" (p. 17). ${ }^{24}$ These concepts refer not only to the ability to discern when intervention is necessary but also the importance of "presence". "Presence" was a feature of exemplary midwifery practice in Kennedy's Delphi study in the US. ${ }^{23}$ Like midwives participating in our study, "presence" or "being with" women in ordinary ways is understood as an activity that contributes to a sense of calm and normalcy. 
The act of "being present" may involve the midwife undertaking an activity such as knitting or having a cup of tea though the primary purpose of the activity is to "be with" the labouring woman and monitor her progress (though this is done in a somewhat covert fashion). ${ }^{25}$ This activity highlights the ordinariness of the situation inspiring calm and reassurance; factors understood my midwives, to facilitate physiological birth.

Place impacted on the midwife's capacity to "be with" childbearing women and also their ability to control the environment so as to create an ambience that is facilitative of physiological birth. This is not the first study to identify this as an important aspect of midwifery work. Studies with midwives from New Zealand, ${ }^{26}$ Norway, ${ }^{27}$ Canada, ${ }^{28}$ the US ${ }^{29}$ and Australia ${ }^{11,30}$ describe midwives' perspectives on the most appropriate ambience for labouring women and findings are remarkably consistent. Midwives typically aim to eliminate intrusive distractions by dimming lights, providing ambient music (of the woman's choosing), preventing breaches of privacy, and attempting to manage people in the environment that might be distracting (with unnecessary chatter for example). Midwives recognise that women need a quiet, calm, private and peaceful environment in which to labour effectively and creating such a space (wherever the birth may planned), is an important part of midwifery practice.

What we are only just beginning to acknowledge is the effect this sort of ambience may have on midwives themselves. Midwives in Everly ${ }^{29}$ and Hammond, Foureur, Homer ${ }^{11}$ studies also describe the impact of this ambience on the midwife. Midwives consistently use the word "relaxing" to describe the effect of this ambience on their own physiology. The effect of stress on the health and wellbeing of individuals is well known impacting on physical and mental health ${ }^{31}$ and furthermore, employers acknowledge the negative impact that stress has on employee performance and workplace productivity. This is illustrated by the proliferation of mindfulness and other stress reduction programs in workplaces. ${ }^{32}$ In 
addition, distractions in the workplace ${ }^{33}$ and high levels of stress have been associated with medical error. ${ }^{34}$ It therefore behoves us to consider the way that birthplaces as the workplaces of midwives, can be modified to so as to be less stress-inducing for midwives. The experience of midwives participating in this study suggests that birth room ambience and workplace culture rather than significant structural or architectural renovations are priorities.

Our study drew on the perspectives of midwives who work across a variety of birthplaces in two countries though they are clearly comfortable working in primary birth settings. This is both a strength and a weakness of this study. The understanding of childbirth shared by the midwives was shaped by their experiences practising in these different environments and they are not as highly acculturated to the labour ward as other midwives may be. This allows them to notice the impact of aspects of the birth environment that may go un-noticed by those who work solely in one setting. These midwives felt more relaxed in primary birth environments and this may not be the experience of midwives who are not comfortable practicing in these environments or working with the same level of autonomy as these midwives. We recognise that this is a small study and included midwives who are probably rare - those who work across all three settings concurrently. However, this cohort provided a unique opportunity to study perceptions of place in a sample of midwives who could articulate the differences of place and were not merely 'different midwives'. Equally, it is interesting that the perceptions across the two countries were the same suggesting that these perceptions have an element of universality. Focus groups exploit the potential of group dynamics and ideally include 8-12 participants. In this study, two to four midwives participated in each focus group and while good discussions were held, the added benefits that a larger group might bring to the discussion, were not realised. 


\section{Conclusion}

While midwives work to the same practice principles in all settings, birthplace nonetheless, has a significant impact on their practice and the way they feel. Creating an ambience appropriate for birth is an important part of their role. This includes dim lighting, ensuring privacy, managing distractions and providing a calm, reassuring midwifery presence. Workplace culture in the labour ward prevents midwives from being able to be with women in this way as it encourages "busy-ness" and surveillance of midwifery practice. Midwives feel more relaxed and able to focus on the woman in settings that allow them to be with women in ordinary ways. The same environment that is most conducive to birth is also conducive to safe midwifery practice. Given the excellent outcomes of midwifery led care we need to focus on ways of ensuring that all birthplace settings are conducive to midwifery practice.

\section{Acknowledgements}

We thank the midwives who kindly agreed to participate. We acknowledge and thank Elizabeth Nicholl who assisted with data collection in Australia and Linda Wylie who undertook the ethics approvals and data collection in the UK. This project has not received any financial support from any individual or organisation. 


\section{References}

1. Birthplace in England Collaborative Group. Perinatal and maternal outcomes by planned place of birth for healthy women with low risk pregnancies: the Birthplace in England national prospective cohort study. BMJ. 2011-11-24 00:00:00 2011;343.

2. Miller S. First birth at home or in hospital in Aotearoa/New Zealand: Intrapartum midwifery are and related outcomes. Wellington, New Zealand, Victoria University of Wellington; 2008.

3. Davis D, Baddock S, Pairman S, et al. Planned Place of Birth in New Zealand: Does it Affect Mode of Birth and Intervention Rates Among Low-Risk Women? Birth. 2011;38(2):111-119.

4. Monk A, Tracy M, Foureur M, Grigg C, Tracy S. Evaluating Midwifery Units (EMU): a prospective cohort study of freestanding midwifery units in New South Wales, Australia. BMJ Open. October 1, 2014 2014;4(10).

5. Hodnett ED, Downe S, Walsh D. Alternative versus conventional institutional settings for birth. Cochrane Database of Systematic Reviews. 2012(Issue 8. Art. No.: CD000012).

6. Wong N, Browne J, Ferguson S, Taylor J, Davis D. Getting the first birth right: A retrospective study of outcomes for low-risk primiparous women receiving standard care versus midwifery model of care in the same tertiary hospital. Women and Birth.

7. Davis D, Walker K. The corporeal, the social and space/place: exploring intersections from a midwifery perspective in New Zealand. Gender, Place \& Culture: A Journal of Feminist Geography. 2010;17(3):377-391.

8. Stenglin M, Foureur M. Designing out the Fear Cascade to increase the likelihood of normal birth. Midwifery. 2013;8(29):819-825.

9. Paul J. Healthy workplace design for healthcare staff. Design \& Health. IV. Future Trends in Healthcare Design. International Academy for Design and Health, Huddinge. 2006:43-52.

10. Symon A, Paul J, Butchart M, Carr V, Dugard P. Maternity unit design study part 4: midwives' perceptions of staff facilities. British Journal of Midwifery. 2008;16(4):228-231. 
11. Hammond A, Foureur M, Homer CSE. The hardware and software implications of hospital birth room design: A midwifery perspective. Midwifery. 2014;30(7):825-830.

12. Ng JL, Johnson A, Nguyen H, Groth M. Workplace Culture Improvements: A Review of the Literature. A report for the Workforce Planning and Development Branch of the NSW Ministry of Health. Sydney, Australia: University of NSW and University of Sydney;2014.

13. O'Connell R, Downe S. A metasynthesis of midwives' experience of hospital practice in publicly funded settings: compliance, resistance and authenticity. Health: November 1, 2009 2009;13(6):589-609.

14. Keating A, Fleming VEM. Midwives' experiences of facilitating normal birth in an obstetric-led unit: a feminist perspective. Midwifery. 2009;25(5):518-527.

15. Kennedy HP, Grant J, Walton C, Shaw-Battista J, Sandall J. Normalizing Birth in England: A Qualitative Study. Journal of Midwifery \& Women's Health. 2010;55(3):262-269.

16. Walsh D. Subverting the assembly-line: Childbirth in a free-standing birth centre. Social Science \& Medicine. 2006;62(6):1330-1340.

17. Sandelowski M. Focus on Research Methods. Whatever Happened to Qualitative Description? Research in Nursing and Health. 2000;23:34-40.

18. Giorgi A. description versus interpretation- cometing alternative strategies for qualitative research. J. Phenomenol. Psychol. Fal 1992;23(2):119-135.

19. Krueger R, Casey M. Focus groups: a practical guide for applied research. 3rd ed. Thousand Oaks: Saga. 3rd ed. Saga: Thousand Oaks; 2000.

20. Jayasekara R. Focus groups in nursing research: methodological perspectives Nursing Outlook. 2012;60:411-416.

21. Creswell J. Research Design. Qualitative, Quantitative and Mixed Methods Approaches. California: Sage Publications; 2003.

22. Sandall J, Soltani H, Gates S, Shennan A, Devane D. Midwife-led continuity models versus othe rmodels of care for childbearing women. Cochrane Database of Systematic Reviews. 2015 (Issue 9. Art. No.: CD004667. ).

23. Kennedy HP. A Model Of Exemplary Midwifery Practice: Results Of A Delphi Study. The Journal of Midwifery \& Women' s Health. 2000;45(1):4-19. 
24. Leap N. The less we do the more we give. In: Kirkham M, ed. The midwifemother relationship. 2nd ed. Hampshire, UK: Palgrave Macmillan; 2010:17-36.

25. Kennedy HP, Shannon MT. Keeping Birth Normal: Research Findings on Midwifery Care During Childbirth. Journal of Obstetric, Gynecologic, \& Neonatal Nursing. 2004;33(5):554-560.

26. Davis DL, Walker K. Case-loading midwifery in New Zealand: making space for childbirth. Midwifery. 2010;26(6):603-608.

27. Blix E. Avoiding disturbance: Midwifery practice in home birth settings in Norway. Midwifery.27(5):687-692.

28. Bourgeault IL, Sutherns R, MacDonald M, Luce J. Problematising public and private work spaces: Midwives' work in hospitals and in homes. Midwifery.28(5):582-590.

29. Everly MC. Facilitators and Barriers of Independent Decisions by Midwives During Labor and Birth. Journal of Midwifery \& Women's Health. 2012;57(1):49-54.

30. Seibold C, Licqurish S, Rolls C, Hopkins F. 'Lending the space': Midwives perceptions of birth space and clinical risk management. Midwifery.26(5):526531.

31. Mollart L, Skinner VM, Newing C, Foureur M. Factors that may influence midwives work-related stress and burnout. Women and Birth. 2013;26(1):26-32.

32. Aikens KA, Astin J, Pelletier KR, et al. Mindfulness Goes to Work: Impact of an Online Workplace Intervention. Journal of Occupational and Environmental Medicine. 2014;56(7):721-731.

33. Mahmood A, Chaudhury H, Valente M. Nurses' perceptions of how physical environment affects medication errors in acute care settings. Applied Nursing Research. 2011;24(4):229-237.

34. Hayashino Y, Utsugi-Ozaki M, Feldman MD, S F. Hope Modified the Association between Distress and Incidence of Self-Perceived Medical Errors among Practicing Physicians: Prospective Cohort Study. PLoS ONE 2012;7(4):e35585. 